\title{
COOPERAÇÃO, ESTRATIFICAÇÃO E PERFIL DA PESQUISA EM ESTRATÉGIA NO BRASIL
}

\section{1- Luciano Rossoni*}

Doutor em Administração pela Universidade Federal do Paraná - UFPR, Curitiba/PR, Brasil.

Professor do Programa de Mestrado e Doutorado em Administração da Universidade Positivo - UP, Curitiba/PR, Brasil. Irossoni@gmail.com

http://lattes.cnpq.br/3158341266410026

\section{2- Edson Ronaldo Guarido Filho}

Doutor em Administração pela Universidade Federal do Paraná - UFPR, Curitiba/PR, Brasil.

Professor do Programa de Mestrado e Doutorado em Administração da Universidade Positivo - UP, Curitiba/PR, Brasil. edson.guarido@gmail.com

http://lattes.cnpq.br/2435628775717990

\section{3- Karine Francisconi}

Mestre em Administração pela Universidade Federal do Paraná - UFPR, Curitiba/PR, Brasil.

Professora da Universidade Positivo - UP, Curitiba/PR, Brasil.

k.francisconi@gmail.com

http://lattes.cnpq.br/6088333860819216

\section{4- José Bonfim Albuquerque Filho}

Doutorando em Administração pela Universidade Federal do Paraná - UFPR, Curitiba/PR, Brasil.

mifnob@gmail.com

http://lattes.cnpq.br/0962173505075635 


\title{
COOPERAÇÃO, ESTRATIFICAÇÃO E PERFIL DA PESQUISA EM ESTRATÉGIA NO BRASIL
}

\section{RESUMO}

A análise documental de publicações permite identificar características e tendências expressas em determinada área do conhecimento científico. Tendo em vista a área de Estratégia em Organizações, este estudo analisou 765 artigos, publicados entre 2001 e 2006, nos anais dos EnAnpad e 3Es, objetivando avaliar quantitativamente os delineamentos de pesquisa utilizados e analisar tendências acerca dos padrões de estratificação e cooperação na área. Por meio de procedimentos estatísticos e da técnica multivariada de análise de correspondência múltipla, verificou-se a predominância de uma produção científica voltada para artigos empíricos apoiados primordialmente em estudos de caso e análises quantitativas tipo surveys. Observou-se também que, ao longo do período investigado, houve crescimento da área no que concerne ao número de artigos, autores e instituições, com ocorrência de maior cooperação na produção acadêmica, além de alterações nos padrões de estratificação regional e institucional.

\section{Palavras-Chave}

Estratégia; produção científica; metodologia; cooperação e produtividade acadêmica; administração

\section{COOPERATION, STRATIFICATION AND RESEARCH DESIGN IN STRATEGY STUDIES IN BRAZIL}

\begin{abstract}
The documentary analysis of publications permits to identify scientific production standards associated with characteristics and tendencies expressed in a certain area of knowledge. Considering the area of Strategy in Organizations and its thematic axes, this study analyzed 765 articles published between 2001 and 2006, in the proceedings of EnAnpad's and 3Es in order to evaluate quantitatively the research design mostly used throughout the period. Moreover, tendencies expressed in terms of stratification standards and cooperation in the area were also investigated. The data were treated in a descriptive-quantitative way, through statistic procedures and multivariate technique of analysis of multiple correspondence. The findings indicated a predominance of a scientific production focused on empirical articles, mainly case studies and surveys. Additionally, the results revealed the growth of the area concerning the number of articles, authors and institutions, accompanied by greater cooperation in scientific production and changes regarding the regional and institutional stratification standards.
\end{abstract}

\section{Keywords}

Strategy; scientific production; methodology; cooperation and academic productivity; management 


\section{Introdução}

O campo de pesquisa em Administração no Brasil vem gradativamente apresentando crescimento na quantidade de publicações científicas. Tomando-se, por exemplo, os encontros anuais da Associação Nacional de Pós-graduação e Pesquisa em Administração (EnANPAD), é notável o acréscimo entre os anos de 2001 e 2006 no número de trabalhos aceitos, que passou de 428 para expressivos 848 artigos, praticamente dobrando em volume nos últimos seis anos. Em 2003, foi criado pela ANPAD o Encontro de Estudos em Estratégia (3Es), evento setorial que, seguindo o exemplo do Encontro Nacional de Estudos Organizacionais (EnEO), nascido em 2000, passou a ser realizado cada dois anos com o objetivo de estabelecer maior espaço destinado à produção científica nas áreas e temas contemplados sob o campo da Estratégia em Organizações ${ }^{1}$. Atualmente, das divisões acadêmicas existentes na ANPAD, a área apresenta o maior número de autores associados, totalizando 137 pesquisadores (FACHIN, 2006). Por essa razão e diante de sua importância para o campo da Administração, faz-se significativo compreender as características de sua produção científica a fim de verificar, sobretudo, em que termos o crescimento quantitativo registrado nos últimos anos tem promovido reflexos sobre o conhecimento gerado na área.

Assim, o presente estudo avaliou a produção científica em Estratégia, sob dois enfoques: quantitativamente, visando apresentar um quadro descritivo do volume e características das publicações, e analiticamente, avaliando as transformações que possam ter ocorrido na área em termos de delineamentos preferenciais de pesquisa (tipo, método e estratégia), grau de cooperação entre autores e estratificação entre instituições e unidades federativas. Para tanto, foram selecionados todos os artigos publicados nos EnANPADs de 2001 a 2006 sob a Divisão Acadêmica de Estratégia (ESO), incluindo as temáticas de Estratégia em Organizações, Gestão Internacional e Empreendedorismo e Comportamento Empreendedor, além dos trabalhos publicados nas edições de 2003 e 2005 do 3Es.

Neste artigo, primeiramente, é realizada uma breve revisão de trabalhos que analisaram a produção científica em diferentes disciplinas da área de Administração, especialmente daqueles que se dispuseram a tratar da área de Estratégia. Posteriormente, são apresentados os procedimentos metodológicos que sustentam a análise dos dados, para que na seção seguinte, sejam expostos os resultados, que apontam tanto a expansão da área em termos de autores, artigos e instituições participantes, como para maior diversidade institucional e cooperação na área. Por fim, conclui-se apontando aspectos relacionados às tendências da área e sugestões para futuras pesquisas.

\section{Produção Científica em Administração e Estratégia}

A avaliação da produção científica brasileira teve início com o trabalho desenvolvido por Machado-daSilva, Cunha e Amboni (1990) que analisaram artigos da área de Organizações publicados entre 1985 e 1989. Na época, os autores constataram que os estudos careciam de melhor desenvolvimento teórico, estando apoiados predominantemente em referências estrangeiras, além de comumente apresentarem pouco rigor metodológico nas estratégias de pesquisa adotadas. Diante de resultados como esses, os autores chamaram a atenção para o fato de, ainda que tenha ocorrido crescimento quantitativo da produção científica na área de organizações, qualitativamente pouco se havia avançado.

Bertero e Keinert (1994), por sua vez, avaliaram a evolução da produção científica brasileira na área de Análise Organizacional, entre 1961 e 1993. Complementarmente ao constatado anteriormente por Machado-da-Silva, Cunha e Amboni (1990), os autores apontaram a multidisciplinaridade presente nas variáveis de pesquisa utilizadas na área, as quais derivavam de diferentes áreas do conhecimento, especialmente da Sociologia e Psicologia, além da Estratégia, Ciência Política e Comunicação. A despeito desse aspecto, os autores concluíram ainda que a produção científica priorizou a replicação e divulgação de idéias desenvolvidas por escolas estrangeiras, com trabalhos predominantemente acadêmicos, isolando a administração do processo de gestão organizacional.

No ano seguinte, Vergara e Carvalho Jr. (1995) avaliaram o tipo, a origem e o padrão de citações da produção científica brasileira na área de organizações. Os autores concluíram, assim como Bertero e Keinert (1994), que, nos trabalhos nacionais, há predominância de material bibliográfico (livros e artigos) de natureza estrangeira, com ainda mínima referência a autores brasileiros.

Após a publicação desses trabalhos, estudos com objetivo de avaliar a produção científica brasileira tornaram-se mais freqüentes no meio acadêmico. Foram realizadas pesquisas que se dedicaram ao campo da Administração de uma forma ampla (p. ex. BERTERO; CALDAS; WOOD JR., 1998; FLEURY, 2003), ou que, 
de modo mais específico, dedicaram atenção a diferentes disciplinas, como Organizações ( $p$. ex. RODRIGUES; CARRIERI, 2001), Estratégia (p. ex. BERTERO; VASCONCELOS; BINDER, 2003; BIGNETTI; PAIVA, 2002; PEGINO, 2005), marketing (p. ex. FROEMMING et al., 2000; VIEIRA, 2003), Recursos Humanos (p. ex. CALDAS; TONELLI; Contabilidade e Finanças (p. ex. CAMARGOS; COUTINHO; AMARAL, 2005), e Administração da Ciência e Tecnologia (p. ex. ROSSONI; FERREIRA JR; HOCAYEN-DA-SILVA, 2009 ).

Seguindo essa tendência, Bignetti e Paiva (2002) analisaram estudos nacionais em Administração Estratégica entre os anos de 1997 e 2001 . Dentre os aspectos por eles discutidos está a constatação de modismos na área, identificados em determinados momentos pela preponderância de referências a determinadas obras traduzidas para o português. Além disso, chamaram atenção para o predomínio anglosaxão indiscutível nos estudos, especialmente de autores norte-americanos, cujo crédito recebido nos artigos era muito maior do que o dos nacionais. Por exemplo, os autores identificaram que apenas um único autor estrangeiro recebeu mais citações no período pesquisado do que o somatório das citações direcionadas a pesquisadores brasileiros. Bignetti e Paiva (2002) relacionaram esse resultado a pouca importância dada aos trabalhos e modelos desenvolvidos no Brasil, devido à posição colonizada dos pesquisadores brasileiros em face dos estudos estrangeiros. Os autores também notaram o número relativamente pequeno de pesquisadores que compunham a área, sugerindo que esse aspecto poderia ser decorrência da pouca integração entre eles, que também possuiriam dificuldades em se dedicar exclusivamente à pesquisa.

Bertero, Vasconcelos e Binder (2003) desenvolveram uma avaliação da produção científica em Estratégia entre os anos de 1991 e 2002, analisando: i) critérios temáticos; ii) metodologias; iii) número de autores; e iv) vinculações institucionais dos autores. De acordo com esse estudo, no que concerne à temática apresentada nos textos da área, destacaram-se fundamentos organizacionais em 18,2\% dos artigos, Porter e fundamentos econômicos em 14,9\%, planejamento estratégico e processo decisório em $8,3 \%$ dos trabalhos cada e recursos e competências em $6,3 \%$ dos artigos. Metodologicamente, $66,3 \%$ dos artigos analisados eram estudos empíricos, compostos por 43,2\% de estudos qualitativos, $19,1 \%$ quantitativos e $4 \%$ mistos. Já dentre os $28,4 \%$ enquadrados como teóricos, $19,8 \%$ deles consistiam em ensaios teóricos, 5,6\% estavam ligados à proposição de modelos de gestão e 3\% eram revisões teóricas. Segundo os autores, porém, a produção científica da área não se refletia na prática das empresas, apresentando poucos resultados que pudessem servir como medida de desempenho para o meio empresarial. Os autores constataram, ainda, que a maioria dos artigos foi produzida por apenas um ( $43,9 \%$ ) ou dois autores $(39,6 \%)$.

Já Pegino (2005) analisou a pesquisa em Administração Estratégica a partir das bases epistemológicas utilizadas nos trabalhos publicados no EnANPAD de 2000 a 2004 . Dentre as abordagens epistemológicas, $41 \%$ dos estudos foram classificados como positivistas, 3\% como fenomenológicos, $7 \%$ funcionalistas e nenhum como dialético ou teórico-crítico. Ainda, para o autor, o estado da arte da pesquisa em Estratégia no Brasil é pouco privilegiado por contribuições teóricas.

Mais recentemente, Rossoni e Guarido Filho (2007) discutem a cooperação entre instituições de ensino e pesquisa no campo da estratégia, avaliando sua implicação sobre a estratificação da produção de conhecimento nessa área, além de seu impacto da produtividade. Segundo os autores, o campo da pesquisa de estratégia em organizações do Brasil, em nível institucional, apresenta uma estrutura de cooperação do tipo small worlds, em que mesmo apresentando grande número de instituições, elas estão aninhadas em grupos locais, ligadas à maioria das instituições em qualquer outro grupo no campo por pequeno número de passos. Em termos de estratificação da produção científica, Rossoni e Guarido Filho (2007) observaram que a área de estratégia é condicionada pela localidade, além de apresentar uma configuração do tipo centro-periferia, cujas organizações mais profícuas tendem a se relacionar mais entre si do que com as demais. Por sua vez, as instituições com menor produção apresentam pouca cooperação entre si, tendendo a se relacionar mais com as instituições mais profícuas (centro). Corroborando tais resultados, os autores verificaram que aquelas instituições que mais colaboram também são as mais produtivas, concluindo que existe relação entre produção científica e estrutura de relações.

Por fim, nessa mesma linha de análise, porém avaliando os grupos de pesquisadores da área de estratégia, Machado-da-Silva e Rossoni (2007) procuraram verificar como o nível de coesão estrutural dos pesquisadores da área de estratégia no Brasil, condicionou a construção do conhecimento científico entre os anos de 1997 e 2005. Os autores observaram que os trabalhos publicados na área foram condicionados, em termos de temas, pelos agrupamentos formados em momentos anteriores. Dessa forma, encontraram alto grau de heterogeneidade dentro dos agrupamentos, mesmo havendo ocorrência de heterogeneidade 
no campo como um todo. Para Machado-da-Silva e Rossoni (2007), tais resultados indicam que a imersão em grupos sociais condiciona o que é legitimamente aceito no campo da pesquisa em estratégia, implicando sistemas de significados potencialmente distintos em cada agrupamento, mas preservando certo nível de comensurabilidade entre diferentes integrantes do campo.

Os diferentes estudos mencionados indicam preocupação crescente nos últimos anos do cientista da Administração em voltar-se para "si mesmo", analisando criticamente a produção acadêmica, no sentido de confrontar qualidade, rigor, relevância e originalidade com o evidente crescimento quantitativo. De modo agregado, os resultados não favorecem a produção nacional, ainda muito apegada ao referencial estrangeiro, pouco cooperativa e carente de contribuições teóricas. Com o propósito de verificar até que ponto esse quadro se alterou nos últimos anos, seguem os procedimentos metodológicos que nortearam o presente estudo.

\section{Procedimentos Metodológicos}

O delineamento do presente estudo pode ser classificado como descritivo-quantitativo, uma vez que busca descrever características e relacionamentos existentes no processo de produção científica da área de Estratégia, utilizando-se, para tanto, um conjunto de categorias analíticas, mensuradas objetivamente e apresentadas, em grande medida, sob a forma de resultados quantificáveis (NEUMAN, 1997; SELLTIZ et al., 1974). Para isso, optou-se pela estratégia de pesquisa documental, caracterizada, segundo Moreira (2005), como um processo de levantamento, verificação e interpretação de documentos que, no presente estudo, compreendeu o conjunto de artigos publicados ao longo dos anos de 2001 a 2006 nas áreas de Estratégia em Organizações, Gestão Internacional e Empreendedorismo, hoje reunidas como Divisão Acadêmica do Encontro Anual da ANPAD (EnANPAD), além daqueles artigos que fizeram parte dos anais das edições de 2003 e 2005 do Encontro de Estudos em Estratégia (3Es).

Os dois eventos foram selecionados por possuírem classificação "A" no sistema Qualis da Capes (Coordenação de Aperfeiçoamento de Pessoal de Nível Superior) e por representarem importantes espaços de comunicação acadêmica na área. Compreenderam a população desse estudo, 575 artigos dos Encontros Nacionais da ANPAD e 190 dos 3Es, totalizando 765 publicações na área de Estratégia. Desse montante, 517 enquadraram-se no eixo temático de Estratégia em Organizações, 136 em Gestão Internacional e 112 em Empreendedorismo e Comportamento Empreendedor ${ }^{2}$. Observa-se que nos anos iniciais não há artigos da temática de Empreendedorismo, criada somente em 2003.

Cada artigo, além de ter registrado seus dados de autoria, foi classificado de acordo com as seguintes categorias de análise: (1) número de artigos publicados por período, evento e eixo temático; (2) tipo de pesquisa (teórica ou teórico-empírica); (3) método de pesquisa (qualitativo, quantitativo ou multimétodo); (4) estratégias qualitativas e quantitativas de pesquisa; (4) instituição de origem dos autores; (5) unidade federativa de origem dos autores.

Esses dados foram dispostos em valores absolutos e percentuais, calculados anualmente ou com base nos períodos de 2001 a 2003 e de 2004 a 2006. A escolha por triênios ocorreu tanto para facilitar o processo de interpretação e análise, como para diminuir a oscilação no número de artigos publicados no decorrer do tempo ocasionada pelo fato de o 3Es ser realizado somente bianualmente. Além disso, após a avaliação do padrão de cooperação e das tendências relacionadas ao tipo, método e estratégia de pesquisa no decorrer dos anos, a opção por reunir os artigos em dois períodos mostrou-se mais adequada, seguindo o critério de maior similaridade entre os anos agrupados.

Após a classificação dos artigos selecionados, os dados foram tabulados e analisados com o apoio do programa Microsoft EXce ${ }^{\circledR}$ e SPSS ${ }^{\circledR}$. As análises realizadas procuraram descrever a área com relação às categorias analíticas mencionadas acima e comparar como os padrões de publicação se comportaram ao longo de diferentes períodos tanto em termos do delineamento dos estudos, quanto em relação aos aspectos ligados à produtividade e cooperação entre autores. Complementarmente, procurou-se ainda avaliar a estratificação da produção na área em termos de instituições e unidades federativas (UF).

Algumas observações devem ser destacadas em relação à condução das análises. A média de autores por artigo foi comparada entre períodos, tipo de pesquisa, método empregado e eixo temático. Para isso foram utilizados o Teste T e o Teste ANOVA com Post Hoc de Tukey. Para avaliar a proporção de artigos por tipo e método de pesquisa entre os períodos ou entre os eixos temáticos foi utilizado o Teste $X^{2}$ (Qui- 
quadrado). Para a contagem das instituições mais prolíficas e do número de artigos por UF, levou-se em conta somente a filiação do primeiro autor dos artigos.

No que diz respeito à pretensão de se verificar se algumas instituições apresentam enfoque mais acentuado em relação às demais quanto a eixos temáticos, tipo ou método de pesquisa adotado, realizouse análise de correspondência múltipla. Por se tratar de categorias de análise de caráter qualitativo, não possibilitando a utilização de testes paramétricos, considerou-se adequada a aplicação desse procedimento. Além disso, essa técnica permite avaliar a interdependência das variáveis e objetos por meio de escalonamento em diversas dimensões (HAIR et al., 2005), o que converge com o objetivo de diferenciar as instituições, com base nas dimensões mencionadas acima. Para tanto, foram selecionados somente os trabalhos daquelas que publicaram mais do que 10 artigos no período, o que resultou em 20 instituições que juntas produziram 566 artigos (74\% do total da produção da área). Essa redução foi necessária para que se pudesse viabilizar a análise com um número restrito de variáveis, representadas no caso pelas 20 instituições. Para não haver interferência da classificação das instituições no escalonamento, essa categoria foi tratada como variável passiva, que, segundo Carvalho (2004), é um procedimento usado, quando se quer entender a relação de uma variável com as demais, mas, por motivações teóricas ou conceituais, não se utiliza para a composição da análise de correspondência. No tocante à classificação das instituições de acordo com as dimensões identificadas, foram seguidos os procedimentos estabelecidos por Carvalho (2004), em que se levam em conta os escores (eigenvalues) das variáveis e das categorias.

Com base nesses procedimentos metodológicos, apresentam-se os resultados da análise da produção científica na área de Estratégia no período de 2001 a 2006, primeiramente por meio da descrição quantitativa de indicadores de produção nos eventos estudados e, depois, a partir das características dos delineamentos de pesquisa utilizados, deixando-se para o final a análise de padrões de cooperação e estratificação por instituição e unidade federativa.

\section{A Pesquisa em Estratégia}

Um primeiro aspecto que pode ser obtido a partir do levantamento dos dados no período é, especialmente após o ano de 2003, o crescimento da área de Estratégia, embora desigual entre os eixos temáticos conforme se apresenta na Tabela 1. Essa consideração revela pelo menos duas informações: (i) o volume total de artigos sofreu aumento, mas se concentrou principalmente no eixo temático de Estratégia em Organizações; e (ii) esse incremento se deveu ao número de trabalhos publicados nos 3 Es. No caso específico do eixo de Estratégia em Organizações, pouco mais da metade das publicações do período ocorreram por meio do 3Es, indicando sua importância na divulgação do conhecimento desenvolvido por acadêmicos da área, em convergência com seu papel enquanto espaço de comunicação acadêmica. Observou-se também que nas duas edições do 3 Es houve grande concentração dos artigos publicados em torno do eixo temático Estratégia em Organizações, ocasionando maior crescimento desse eixo que os demais.

Tabela 1 - Artigos publicados na área de Estratégia de 2001 a 2006.

\begin{tabular}{cccccccccc}
\hline \multirow{2}{*}{ Eixo Temático } & 2001 & 2002 & \multicolumn{2}{c}{2003} & 2004 & 2005 & 2006 & Total \\
& EnA & EnA & EnA & 3Es & EnA & EnA & 3Es & EnA & \\
\hline Estratégia & 58 & 55 & 49 & 87 & 52 & 70 & 86 & 60 & 517 \\
Gestão Internacional & 10 & 17 & 22 & 1 & 26 & 26 & 7 & 27 & 136 \\
Empreendedorismo & 0 & 0 & 25 & 4 & 33 & 22 & 5 & 23 & 112 \\
\hline Total & 68 & 72 & 96 & 92 & 111 & 118 & 98 & 110 & \\
Total Ano & 68 & 72 & 188 & 111 & 216 & & 110 & 765 \\
\hline *EnA = EnANPAD & & & & & & & & &
\end{tabular}

No entanto, mesmo não havendo hierarquização de eixos temáticos dentro da área por parte da ANPAD, as temáticas de Empreendedorismo e de Gestão Internacional não acompanharam o crescimento apresentado pela área como um todo, o que se pode perceber pelo pouco acréscimo de artigos com a criação do 3Es. Fica a indagação sobre as razões que produziram esse efeito. É possível que sua produção tenha migrado para outras áreas ou eventos ou, ainda, que sofram influência do número reduzido de linhas de pesquisa nessas perspectivas (ROSSONI, 2006). Além disso, pode-se considerar que o interesse pelos fenômenos da internacionalização e empreendedorismo seja ainda recente no meio acadêmico, já que até 
há pouco tempo eram discutidos apenas perifericamente em algumas disciplinas da Administração. Somase, especificamente, no caso da Gestão Internacional, o fato de o processo de internacionalização das organizações nacionais estar em seu estágio inicial de desenvolvimento, de modo que o acesso ao mercado internacional permanece restrito à grande massa de empresas. Por outro lado, a limitação em termos do número de artigos pode ser decorrente da delimitação desses dois eixos temáticos, mais específicos quanto ao seu foco de estudos do que a temática Estratégia em Organizações, que permite englobar uma diversidade maior de unidades de análise e enfoques.

Outro indicador representativo do crescimento da área é a quantidade de autores que participam anualmente de publicações. Afora o ano de 2006, que apresentou redução nesse número em relação ao anterior, desde 2001 nota-se crescimento gradual, partindo de 68 e alcançando 399 autores com publicações na área no ano de 2005, quando ocorreu a segunda edição do 3Es. Esse número, no entanto, embora possa indicar maior incidência de participantes na produção de trabalhos, não permite afirmar que represente a totalidade de pesquisadores que efetivamente atuam na área. Ademais, de acordo com Braun, Glänzel e Schubert (2001), avaliou-se a proporção de autores com atuação contínua no período, em comparação com aqueles que estariam entrando, se retirando ou apenas de passagem pela área de Estratégia. Registraram-se, para o ano de 2004, 46 entrantes (autores que não possuíam publicação anterior até o ano em questão e publicaram posteriormente) e 29 continuantes (que já haviam publicado antes e continuaram publicando em anos seguintes), e 26 e 34, respectivamente em 2005 e 2006 , indicando maior permanência de autores na área. Evidentemente, diante do período limitado desse estudo, esses dados devem ser lidos com o devido cuidado. No entanto eles indicam que somente uma reduzida parte dos autores se dedica à pesquisa em Estratégia de forma consistente.

Além das diferenças relativas ao número de autores e artigos publicados por cada eixo temático, o crescimento da área de Estratégia possibilitou também comparar a produção científica em termos de delineamentos de pesquisa e padrões de cooperação e produtividade ao longo dos anos. Suas diferenças e similaridades são expostas a seguir.

Quanto ao tipo de pesquisa, observou-se que a área de Estratégia privilegia estudos de caráter empírico, com $83 \%$ do total de artigos publicados dessa natureza (vide Tabela 2). Em contraponto, o número de ensaios teóricos apresenta percentual bastante inferior, representando somente $17 \%$ do total de artigos do período. Em comparação com outras áreas da Administração, esse percentual é similar ao de finanças (CAMARGOS; COUTINHO; AMARAL, 2005) e marketing (PROENÇA; LOPES; MEUCCI, 2005), que também apresentam predominância de estudos empíricos. Deve-se ressaltar que a discussão sobre Estratégia sempre foi valorizada por organizações, principalmente aquelas que contratavam serviços de consultores, muitos desses oriundos do meio acadêmico, fato que aproxima a temática da atividade empresarial, refletindo-se em maior quantidade de estudos empíricos (MACHADO-DA-SILVA; VIZEU, 2007).

A diminuição na proporção de ensaios teóricos reforça a tendência já apontada em outras áreas da Administração (CALDAS; TONELLI; LACOMBE, 2002; TONELLI et al., 2003). Em Estratégia, o número de artigos teóricos diminuiu significativamente (Qui-quadrado $=5,140, p=0,023$ ), de 20\% para 14\%, na comparação entre os dois períodos estudados, conforme mostra a tabela 2.

Tabela 2 - Tipo e Método de Pesquisa.

\begin{tabular}{|c|c|c|c|c|c|c|}
\hline \multirow{2}{*}{$\begin{array}{l}\text { Tipo de Pesquisa } \\
\text { Ensaio Teórico }\end{array}$} & \multicolumn{2}{|c|}{ 2001-2003 } & \multicolumn{2}{|c|}{ 2004-2006 } & \multicolumn{2}{|c|}{ Total } \\
\hline & 66 & $20 \%$ & 61 & $14 \%$ & 127 & $17 \%$ \\
\hline Estudo Empírico & 262 & $80 \%$ & 376 & $86 \%$ & 638 & $83 \%$ \\
\hline Total & 328 & & 437 & & 765 & \\
\hline \multicolumn{7}{|c|}{ Método de Pesquisa (Estudos Empíricos) } \\
\hline Qualitativo & 175 & $67 \%$ & 215 & $57 \%$ & 390 & $61 \%$ \\
\hline Quantitativo & 72 & $27 \%$ & 141 & $38 \%$ & 213 & $33 \%$ \\
\hline Multimétodo & 15 & $6 \%$ & 20 & $5 \%$ & 35 & $5 \%$ \\
\hline Total & 262 & & 376 & & 638 & \\
\hline
\end{tabular}

Para Bertero, Caldas e Wood Jr (1998), o volume mais elevado de artigos empíricos pode ser sinal de maior rigor no processo de avaliação de trabalhos, o que indicaria amadurecimento da pesquisa na área, podendo levar à aceitação somente de ensaios com contribuições teóricas relevantes. Por outro lado, pode representar redução no empenho em sistematizar teoricamente os diferentes achados empíricos, já que foi 
possível notar que, desde o ano de 2003, houve reversão de crescimento desse tipo de estudo para proporções bem menores nos anos seguintes, especialmente nos EnANPAD's. Já nos 3Es, embora significativamente menor que os estudos empíricos, sua proporção não é tão reduzida, o que destaca esse evento com relação à divulgação desse tipo de pesquisa.

Diferença significativa entre tipos de pesquisa ocorreu também na comparação entre eixos temáticos (Qui-quadrado $=11,846, p=0,003$ ). Enquanto ensaios teóricos representam 18,7\% das publicações em Estratégia e 17,6\% em Gestão Internacional, não passam de 5,4\% em Empreendedorismo, explicitando a pouca consistência no debate teórico acerca desse tema, já observado por Guimarães (2004) e Reader e Watkins (2006)

No tocante ao método de pesquisa empregado, verificou-se que pouco mais da metade dos artigos publicados na área (390 dos 765) utilizam métodos qualitativos de pesquisa. Artigos de natureza quantitativa representam $28 \%$ do total de artigos e trabalhos que utilizaram tanto métodos qualitativos quanto quantitativos (multimétodos) correspondem a aproximadamente $4,5 \%$ do volume total publicado na área no período estudado. No entanto, mesmo sendo maioria, estudos qualitativos vêm significativamente perdendo espaço para quantitativos (Qui-quadrado $=7,023, p=0,03$ ). Entre os anos de 2001 e 2003 , somente $27,4 \%$ dos artigos utilizaram métodos quantitativos contra 37,5\% na média do período de 2004 a 2006. Essa tendência, ilustrada no Gráfico 1, tem sido valorizada pela coordenação da área da ANPAD, como observado pelo professor Jorge Ferreira da Silva:

A aplicação crescente da metodologia quantitativa de qualidade, elaborada a partir de sólidos construtos com base teórica no estado da arte e utilizando de dados de última geração, além de novas aplicações de inteligência computacional, marcaram as pesquisas teórico-empíricas reportadas nos papers submetidos (ANPAD, 2006, p. 225-226).

Gráfico 1 - Tipo e Método de Pesquisa: evolução entre 2001 e 2006

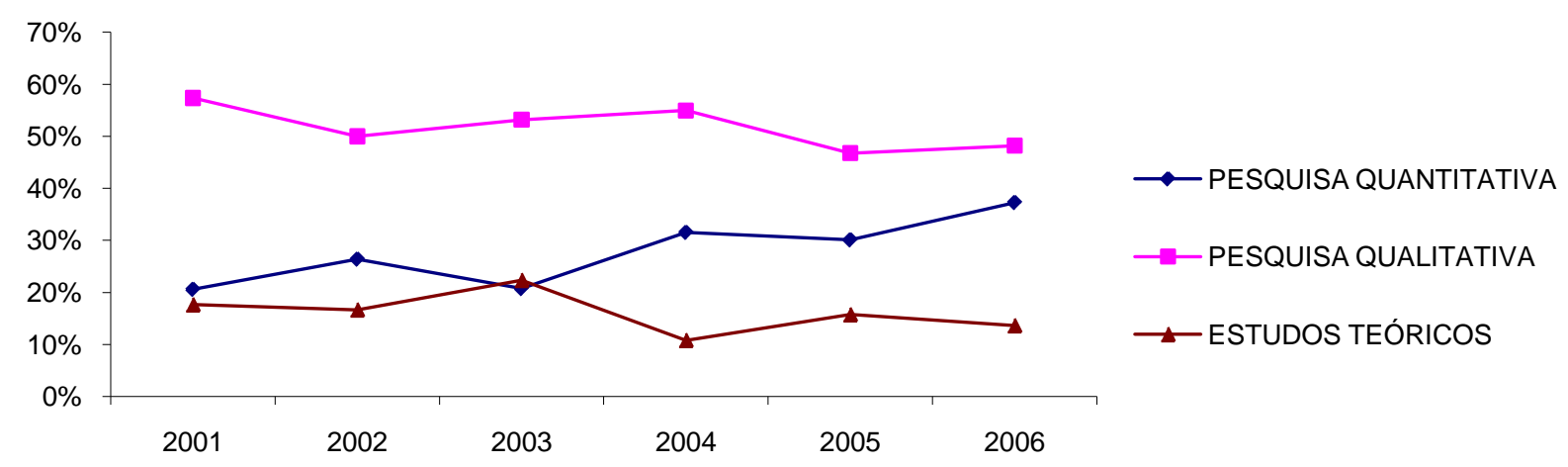

Outros autores entendem que esse incremento de artigos quantitativos acompanha uma tendência internacional e acreditam que tais estudos atendem mais rigorosamente a critérios científicos, como validade e confiabilidade. Por exemplo, Bertero, Vasconcelos e Binder (2003) e Hoppen e Meirelles (2005) afirmam que a ampla utilização de métodos qualitativos expressa a fragilidade e a imaturidade do campo de pesquisa em Administração, não sendo diferente a área de Estratégia. Segundo esses autores, muitos dos estudos realizados na área de Estratégia provavelmente ficam somente na fase exploratória, não evoluindo para a construção de estudos de natureza explicativa, o que provoca, conseqüentemente, descontinuidade no desenvolvimento dos temas nessa área.

Diferença significativa também foi encontrada na comparação entre eixos temáticos com relação ao método de pesquisa (Qui-quadrado $=10,730, \mathrm{p}=0,03$ ). Enquanto Gestão Internacional $\mathrm{e}$ Empreendedorismo contam, respectivamente, com $25 \%$ e $26,6 \%$ de trabalhos apoiados em metodologias quantitativas entre os estudos empíricos publicados nessas áreas, Estratégia em Organizações alcança $37,3 \%$, indicando preferência por este tipo de estudo, se comparado com as demais temáticas. Já com relação aos estudos multimétodos, destaca-se Gestão Internacional com aproximadamente $9 \%$ dos seus trabalhos empíricos, o dobro do índice encontrado na média das publicações dos outros eixos temáticos. Nesse caso, predominam estratégias quantitativas do tipo survey mescladas com pesquisa documental, já 
que a utilização de marcos legais e relatórios governamentais costumam ser fontes relevantes de evidência para trabalhos em Gestão Internacional, o que provavelmente fomentou sua maior utilização.

Com relação às estratégias de pesquisa, entre os 425 estudos de natureza qualitativa, verificou-se predominância dos estudos de caso, presentes em $66 \%$ dos artigos desse conjunto (vide Tabela 3), o que converge com achados nas áreas de Administração da Ciência e Tecnologia (ROSSONI; FERREIRA JÚNIOR; HOCAYEN-DA-SILVA, 2009), Administração Pública e Gestão Social (HOCAYEN-DA-SILVA; ROSSONI; FERREIRA JÚNIOR, 2008) e Sistemas de Informação (HOPPEN et al., 1998). Duas outras estratégias qualitativas também foram amplamente utilizadas: o estudo de campo (15\%) e a pesquisa documental $(9,6 \%)$. Os estudos de campo se utilizam primordialmente de entrevistas na coleta de dados, sendo o material tratado, na maioria das vezes, somente de forma qualitativa. Na maioria dos casos avaliados, artigos que se utilizaram desse método apresentaram pouco rigor metodológico e nenhum tipo de mecanismo de validade e de confiabilidade como, por exemplo, triangulação de fontes de evidência, não se configurando nem como caso, nem como etnografia. Análises de conteúdo, baseadas especialmente no tratamento sugerido por Bardin, também obtiveram algum destaque; mas outras estratégias de pesquisa qualitativas foram utilizadas apenas perifericamente; nenhuma delas atingiu mais que $1 \%$.

Tabela 3 - Estratégias Qualitativas de Pesquisa.

\begin{tabular}{|c|c|c|c|c|c|c|}
\hline \multirow{2}{*}{$\begin{array}{l}\text { Estratégia } \\
\text { Estudo de Caso }\end{array}$} & \multicolumn{2}{|c|}{ 2001-2003 } & \multicolumn{2}{|c|}{ 2004-2006 } & \multicolumn{2}{|c|}{ Total } \\
\hline & 119 & $63 \%$ & 163 & $69 \%$ & 282 & $66 \%$ \\
\hline Estudo de Campo & 27 & $14 \%$ & 37 & $16 \%$ & 64 & $15 \%$ \\
\hline Pesquisa Documental & 27 & $14 \%$ & 14 & $6 \%$ & 41 & $10 \%$ \\
\hline Análise de Conteúdo & 9 & $5 \%$ & 15 & $6 \%$ & 24 & $6 \%$ \\
\hline Pesquisa-ação & 1 & $1 \%$ & 2 & $1 \%$ & 3 & $1 \%$ \\
\hline Etnografia & 3 & $2 \%$ & 1 & $0,4 \%$ & 4 & $1 \%$ \\
\hline \multirow{2}{*}{$\begin{array}{l}\text { Observação Participante } \\
\text { Grounded Theory }\end{array}$} & 2 & $1 \%$ & - & - & 2 & $0,5 \%$ \\
\hline & 1 & $1 \%$ & 1 & $0,4 \%$ & 2 & $0,5 \%$ \\
\hline Outras & 1 & $1 \%$ & 2 & $1 \%$ & 3 & $1 \%$ \\
\hline Total Período & 190 & & 235 & & 425 & \\
\hline
\end{tabular}

Os achados até aqui reforçam o que Bertero, Vasconcelos e Binder (2003) já haviam observado sobre a incipiência metodológica presente nos artigos da área de Estratégia no Brasil, cujos trabalhos empíricos não implicam utilização adequada de várias estratégias de pesquisa, mas o predomínio do estudo de caso. Para eles, "os estudos de caso a rigor são freqüentemente 'exemplos', não atendendo às exigências do protocolo habitual para estudos de caso enquanto pesquisa academicamente válida" $(2003$, p. 57). Ainda segundo os autores, estudos de caso, em geral, são caracterizados como veículos de divulgação e promoção de empresas, ou como suporte técnico para consultorias desenvolvidas na área. Apesar dessa ressalva, não se constatou redução significativa na proporção de estudos de caso entre as pesquisas qualitativas, embora, se observado em relação ao volume total de artigos na área, seu número tenha diminuído, devido ao avanço dos estudos quantitativos.

O mesmo foi constatado na análise das estratégias quantitativas de pesquisa, em que a survey foi predominante, estando presente em $75,4 \%$ dos artigos dessa natureza (vide Tabela 4), coincidindo com o encontrado por Froemming et al. (2000), quando elaboraram um inventário das características metodológicas básicas da produção científica da área de marketing no Brasil, na década de 90. Constatouse também que a maioria das pesquisas desse tipo utilizou pacotes estatísticos para a análise de dados, frequentemente aplicando técnicas de análise multivariada como análise fatorial, análise de clusters, regressão e equações estruturais. Todavia há também outros estudos que aplicaram técnicas estatísticas robustas; mas não podem ser enquadrados como surveys, por não terem feito levantamentos. Esses estudos foram categorizados como análise estatística, sendo a segunda estratégia mais presente com aproximadamente $11 \%$ do total de pesquisas quantitativas. Nesses artigos, a coleta ocorre principalmente em base de dados como, por exemplo, 500 Maiores e Melhores do Guia Exame ${ }^{\circledR}$, Compustat ${ }^{\circledR}$ e Economática ${ }^{\circledR}$. Tal estratégia é cada vez mais freqüente, principalmente em trabalhos que avaliam desempenho de organizações, pois fornecem grande quantidade de casos, sem a necessidade de ir a campo para coleta. Estratégias como análise de séries temporais ou dados em painel, embora presentes em $4,8 \%$ dos artigos de natureza quantitativa, vêm diminuindo cada ano, ao contrário de áreas, como 
Finanças (CAMARGOS; COUTINHO; AMARAL, 2005), em que são utilizadas com maior freqüência. Para as demais estratégias de pesquisa, o que se percebeu foi um acréscimo de estudos que aplicaram análise de redes sociais, modelagem, simulação e experimentos; mas ainda representam pequena parcela, não maior do que $3 \%$ para cada uma delas.

Tabela 4 - Estratégias quantitativas de pesquisa.

\begin{tabular}{|c|c|c|c|c|c|c|}
\hline \multirow{2}{*}{$\frac{\text { Estratégia }}{\text { Survey }}$} & \multicolumn{2}{|c|}{ 2001-2003 } & \multicolumn{2}{|c|}{ 2004-2006 } & \multicolumn{2}{|c|}{ Total } \\
\hline & 67 & $77 \%$ & 120 & $75 \%$ & 187 & $75 \%$ \\
\hline Análise Estatística & 9 & $10 \%$ & 18 & $11 \%$ & 27 & $11 \%$ \\
\hline Análise de Séries Temporais & 6 & $7 \%$ & 6 & $4 \%$ & 12 & $5 \%$ \\
\hline Análise de Redes & 2 & $2 \%$ & 5 & $3,1 \%$ & 7 & $3 \%$ \\
\hline Modelagem e Simulação & 2 & $2 \%$ & 6 & $4 \%$ & 8 & $3,2 \%$ \\
\hline Experimento & 1 & $1 \%$ & 5 & $3 \%$ & 6 & $2 \%$ \\
\hline Outros & - & - & 1 & $1 \%$ & 1 & $0,4 \%$ \\
\hline Total Período & 87 & & 161 & & 248 & \\
\hline
\end{tabular}

Em linhas gerais, com relação à proporção de estratégias de pesquisa qualitativas ou quantitativas presentes nos trabalhos, não foram constatadas diferenças significativas na comparação entre períodos, eixos temáticos ou eventos. Entretanto, foi possível perceber que, no tocante apenas às estratégias quantitativas, existe maior variabilidade delas entre os artigos presentes nos EnANPAD's do que nos $3 E s$ (Qui-quadrado $=16,801, p=0,010$ ).

\section{Cooperação Científica na Pesquisa em Estratégia}

Além dos delineamentos de pesquisa, verificaram-se padrões de cooperação e produção dos autores e instituições. De modo geral, foi possível identificar aumento significativo no grau de cooperação entre autores na produção científica na área de Estratégia ( $t=-5,282, p<0,001)$, especialmente após o ano de 2003, conforme ilustrado no Gráfico 2. Esse padrão foi percebido independentemente do evento em questão e foi mais significativo no eixo temático de Estratégia em Organizações que, ao longo do período de 2001 a 2006, teve sua média de autores por artigo elevada de 1,79 para 2,52. Como se pode notar no Gráfico 2, com base em Braun, Glänzel e Schubert (2001) e em convergência com Rossoni (2006), maior cooperação (calculada pela proporção de autorias por artigo), tem levado à diminuição da produtividade, seja a medida pela contagem (relação entre autorias por autor) ou fracionada (proporção de artigos por autor), indicando maior divisão do trabalho no processo de produção de artigos.

Gráfico 2 - Padrões de Cooperação e Produtividade.

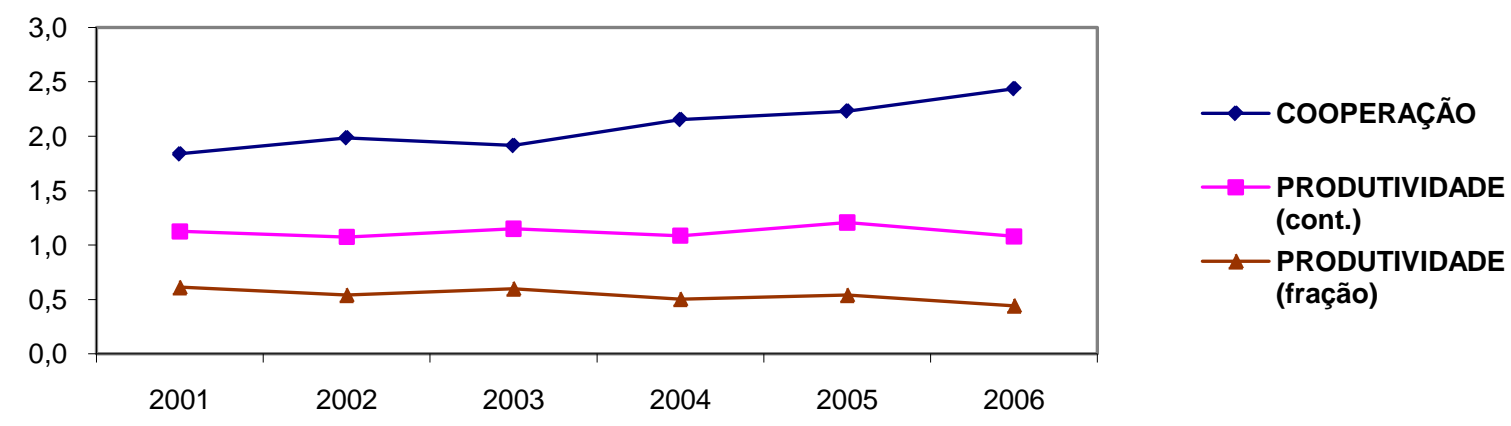

No entanto é interessante notar que, considerando todo o período, ensaios teóricos tendem a apresentar menor número de autores que estudos empíricos, na proporção de 1,78 para 2,18 autores por artigo respectivamente $(t=-4,545, p<0,001)$. Tais resultados são semelhantes aos encontrados por Hocayen-da-Silva, Rossoni e Ferreira Júnior (2008) na área de Administração Pública e apontam a existência de certo grau de concentração em número reduzido de autores na elaboração de trabalhos teóricos na área (14\% do total). Contudo, diferentemente do apontado por outros estudos (HOCAYEN-DA- 
SILVA; ROSSONI; FERREIRA JÚNIOR, 2008; MOODY, 2004; ROSSONI; FERREIRA JÚNIOR; HOCAYEN-DA-SILVA, 2009), não se observou diferença estatística significativa na cooperação, quando comparados os artigos segundo os métodos de pesquisa empregados $(p=0,257)$, mesmo tendo sido possível notar menor cooperação entre autores que publicaram estudos qualitativos. É provável que esse resultado tenha sido influenciado pelo fato da temática de Empreendedorismo apresentar, ao mesmo tempo, maior número de autores por artigo e mais artigos qualitativos. Como esse eixo apresenta poucos autores prolíficos, acaba acarretando número maior de autores na produção de menor quantidade de artigos. Também não foi encontrada diferença significativa entre os eixos temáticos $(p=0,089)$, embora em Estratégia em Organizações tenha havido mudança significativa, quando comparada em bases anuais a cooperação de autores desse eixo temático isoladamente.

\section{Estratificação da Produção}

Para que se pudesse ter um quadro da estratificação e concentração da produção em Estratégia foram analisadas as instituições mais prolíficas da área. Como já ressaltado por Bertero, Caldas e Wood Jr. (1998), o conhecimento científico é estratificado, apresentando centro e periferia, no qual algumas poucas instituições representam maioria da produção científica. Na Tabela 5 é possível visualizar que as três instituições com maior número de artigos no período de 2001 a 2006 estão localizadas no Estado de São Paulo: FGV-SP, USP e Mackenzie. Juntas, elas representam 16,9\% da produção da área. Se tomadas as cinco instituições mais prolíficas, têm-se $26,5 \%$ da produção da área, ou seja, mais de um quarto da produção da área estão concentrados em 3,35\% das 149 instituições identificadas. Embora esse grau de concentração seja elevado, constatou-se queda gradual ao longo dos anos (de 43\% em 2002 para 30\% em 2006), além de ser menor do que o revelado em outras áreas como Administração da Ciência e Tecnologia e Administração Pública, cuja produção das cinco instituições mais prolíficas alcança $36 \%$ e $49 \%$ respectivamente, do publicado nessas áreas.

Tabela 5 - Número de artigos publicados por instituição.

\begin{tabular}{|c|c|c|c|c|c|}
\hline \multicolumn{6}{|c|}{ Período } \\
\hline Instituição & 2001-2003 & 2004-2006 & Total & $\%$ & $\%$ Acumulado \\
\hline Total & 318 & 437 & 755 & $100 \%$ & \\
\hline FGV-SP & 19 & 26 & 45 & $6,0 \%$ & $6,0 \%$ \\
\hline USP & 15 & 29 & 44 & $5,8 \%$ & $11,8 \%$ \\
\hline MACKENZIE & 12 & 28 & 40 & $5,3 \%$ & $17,1 \%$ \\
\hline UFRGS & 19 & 16 & 35 & $4,6 \%$ & $21,7 \%$ \\
\hline UFRJ & 15 & 20 & 35 & $4,6 \%$ & $26,4 \%$ \\
\hline UNISINOS & 15 & 19 & 34 & $4,5 \%$ & $30,9 \%$ \\
\hline PUC-MG & 19 & 14 & 33 & $4,4 \%$ & $35,2 \%$ \\
\hline UFMG & 12 & 19 & 31 & $4,1 \%$ & $39,3 \%$ \\
\hline UFPR & 13 & 17 & 30 & $4,0 \%$ & $43,3 \%$ \\
\hline PUC-PR & 14 & 15 & 29 & $3,8 \%$ & $47,2 \%$ \\
\hline UFPE & 14 & 13 & 27 & $3,6 \%$ & $50,7 \%$ \\
\hline UNIFOR & 11 & 16 & 27 & $3,6 \%$ & $54,3 \%$ \\
\hline UNIVALI & 13 & 13 & 26 & $3,4 \%$ & $57,7 \%$ \\
\hline PUC-RJ & 8 & 15 & 23 & $3,0 \%$ & $60,8 \%$ \\
\hline FGV-RJ & 5 & 16 & 21 & $2,8 \%$ & $63,6 \%$ \\
\hline UEL-UEM & 12 & 9 & 21 & $2,8 \%$ & $66,4 \%$ \\
\hline PUC-SP & 6 & 11 & 17 & $2,3 \%$ & $68,6 \%$ \\
\hline PUC-RS & 5 & 10 & 15 & $2,0 \%$ & $70,6 \%$ \\
\hline UFLA & 4 & 10 & 14 & $1,9 \%$ & $72,5 \%$ \\
\hline Todas as outras & 3 & 6 & 208 & $27,5 \%$ & $100,0 \%$ \\
\hline
\end{tabular}

Acredita-se que parte da concentração de artigos nessas instituições se deva à existência de linhas de pesquisa na área, tamanho do programa de pós-graduação e políticas institucionais de fomento à produção científica, como pode ser o caso da Universidade Mackenzie que, apesar de apresentar menor número de professores do que outras instituições, ampliou sua participação na área de Estratégia nos últimos anos, 
tendo publicado somente menos artigos do que a USP no último período.

Esse movimento de diversificação e de redução da concentração na área de Estratégia pode ser verificado a partir de outros indicadores. Por exemplo, em 2001, o volume publicado por oito instituições representava $50 \%$ da produção da área, quadro que se vem alterando lentamente após 2003, quando o número de instituições subiu para 10 e se vem mantendo em torno desse patamar até o momento. Somando-se esse resultado com a redução da concentração no topo é possível concluir que tem havido maior distribuição de trabalhos entre instituições, diversificando a produção na área em termos da origem institucional dos artigos. Se for observado ainda que o número de instituições participantes na área de Estratégia subiu de 87 para 115 quando comparados os dois períodos de corte, fica mais evidente a tendência de transformação no quadro acadêmico da produção na área.

A área também sofreu mudanças no que se refere à cooperação institucional e à produtividade. Na comparação entre os dois períodos tem-se a elevação da produtividade por instituição na ordem de $19 \%$. Visto que a produtividade por autor vem caindo, conforme Gráfico 2, considera-se que esse incremento de produção seja possivelmente reflexo do maior número de autores na área. Já em termos de colaboração institucional, o que se nota é o aumento do componente principal ${ }^{3}$ da rede institucional nesse último período, com crescimento significativo do número de laços, de 1,33 para 1,93 ( $t=-2,253, p=0,025$ ), ampliando de 44 para 71 instituições que colaboram ativamente entre si por meio de seus acadêmicos na produção de trabalhos. Além disso, a produtividade decrescente do componente principal (de 14,27 para 13,91 artigos por instituição) reforça o aspecto da diversificação apontado acima.

Não somente as diferenças em termos da quantidade de produção científica foram identificadas entre as instituições. Buscando-se investigar se algumas delas tendem a privilegiar determinado eixo temático, tipo ou método de pesquisa, utilizou-se a análise de correspondência múltipla, considerando 20 das mais prolíficas instituições da área e que juntas respondem por cerca de $74 \%$ da produção em Estratégia. Esse procedimento consistiu em criar um espaço topográfico em que os objetos analisados são dispostos de acordo com sua similaridade em relação às variáveis estudadas. No presente estudo, os escores da análise indicaram duas dimensões: a primeira relativa ao tipo de pesquisa, teórica ou empírica, identificada pelo eixo horizontal; e a segunda correspondente ao eixo temático e método de pesquisa, indicada pelo eixo vertical. No espaço bidimensional resultante, enquadraram-se as instituições segundo a predominância em seus estudos das categorias de análise indicadas.

A partir da inspeção visual e da avaliação dos escores das instituições foi possível definir quatro agrupamentos, conforme indicados no Gráfico 3.

. Gráfico 3 - Análise de correspondência múltipla entre eixo temático, tipo e método de pesquisa.

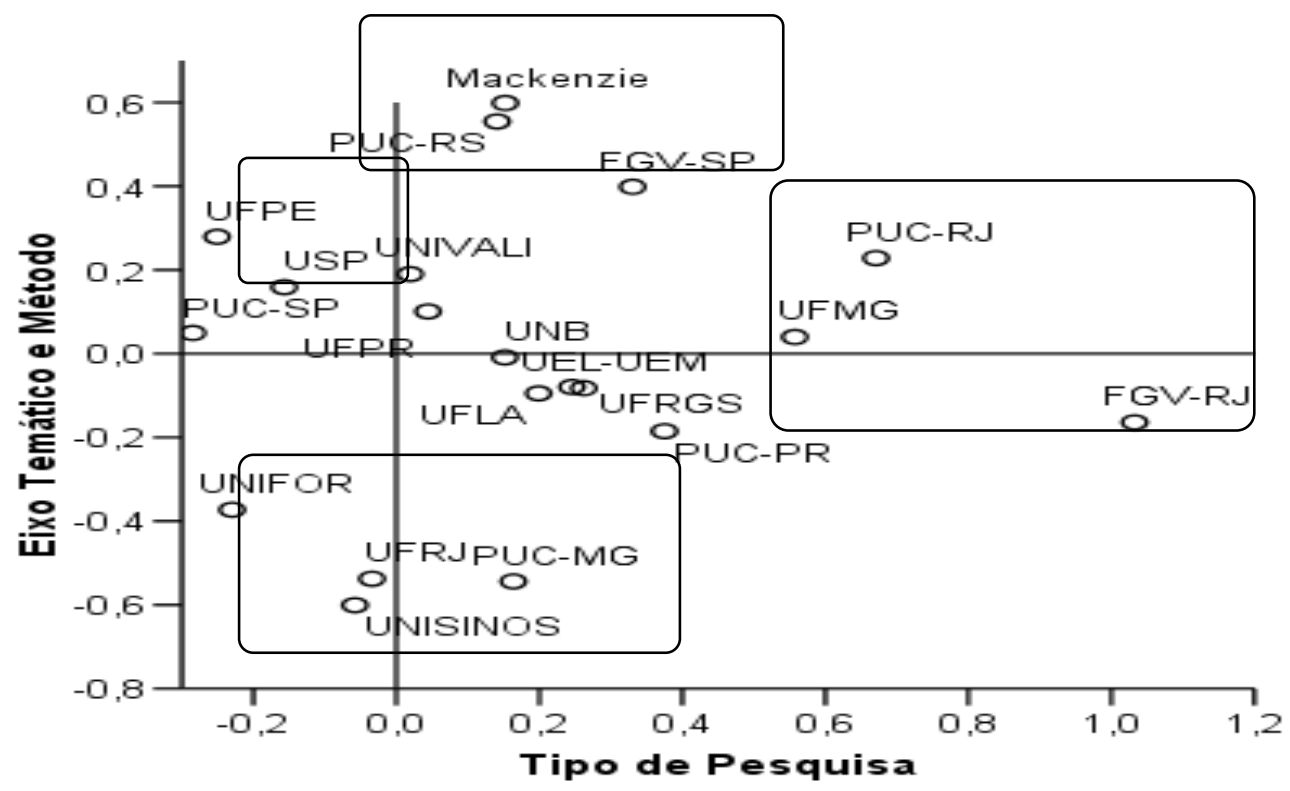

Aquelas instituições não enquadradas em nenhum dos grupos apresentaram pequena discriminação e, por essa razão, tendem a ter o padrão médio da área de Estratégia, caracterizado por maioria de artigos 
empíricos qualitativos. As instituições encontradas nessa situação foram UFPR, UNIVALI, PUC-SP, UEL-UEM, UFRGS, UFLA, UNB E PUC-PR.

O primeiro grupo, composto por PUC-RS, Mackenzie e FGV-SP, localizado na parte superior do gráfico, diferencia-se em relação às outras instituições com relação à proporção de estudos empíricos baseados em métodos quantitativos e orientados principalmente para o eixo temático de Estratégia em Organizações. Isso, no entanto, não significa que possuam maioria de seus artigos de acordo com esse perfil, mas apenas que apresentam, proporcionalmente, mais trabalhos nessas condições do que as outras instituições, de forma geral. Dessas três instituições, vale destacar o trabalho dos pesquisadores da Mackenzie na construção de modelos quantitativos com técnicas avançadas. No caso da FGV-SP, a maioria dos trabalhos na área foi liderada por Flávio Vasconcelos e Luiz Brito, que se vêm dedicando à pesquisa da vantagem competitiva nas organizações.

No quadro situado acima e à esquerda do gráfico, estão UFPE e USP, ambas agrupadas por apresentarem maior percentual de artigos empíricos que a maioria das instituições, além de trabalharem mais freqüentemente com métodos qualitativos que as demais. No caso da UFPE, sua discriminação se dá também por focar a temática de Empreendedorismo com maior destaque

O terceiro grupo de instituições, localizado na parte inferior do gráfico é composto por quatro instituições, UNIFOR, UNISINOS, UFRJ e PUC-MG, que, ao longo do período estudado, produziram proporcionalmente mais artigos sobre a temática de Gestão Internacional do que outras instituições. Além disso, diferenciam-se das demais por apresentarem mais trabalhos apoiados em multimétodos que as demais instituições. É interessante notar que não há nesses programas linhas de pesquisa em Gestão ou Estratégias de Internacionalização, de modo que leve a acreditar que tal resultado decorre da atuação de alguns pesquisadores interessados no tema e organizados em grupos de pesquisa.

Tabela 6 - Número de artigos publicados por unidade federativa

\begin{tabular}{|c|c|c|c|c|c|}
\hline \multicolumn{6}{|l|}{ 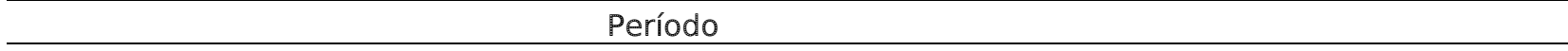 } \\
\hline Unid. Federativa & 2001-2003 & 2004-2006 & Total & $\%$ & $\%$ Acum. \\
\hline Total & 318 & 437 & 755 & $100 \%$ & \\
\hline SP & 70 & 124 & 194 & $25,7 \%$ & $25,7 \%$ \\
\hline MG & 42 & 55 & 97 & $12,8 \%$ & $38,5 \%$ \\
\hline PR & 44 & 46 & 90 & $11,9 \%$ & $50,5 \%$ \\
\hline RJ & 33 & 56 & 89 & $11,8 \%$ & $62,3 \%$ \\
\hline RS & 47 & 42 & 89 & $11,8 \%$ & $74,0 \%$ \\
\hline SC & 23 & 37 & 60 & $7,9 \%$ & $82,0 \%$ \\
\hline CE & 16 & 25 & 41 & $5,4 \%$ & $87,4 \%$ \\
\hline PE & 15 & 15 & 30 & $4,0 \%$ & $91,4 \%$ \\
\hline EXT & 7 & 14 & 21 & $2,8 \%$ & $94,2 \%$ \\
\hline DF & 5 & 6 & 11 & $1,5 \%$ & $95,6 \%$ \\
\hline $\mathrm{BA}$ & 5 & 5 & 10 & $1,3 \%$ & $97,0 \%$ \\
\hline AM & 3 & 3 & 6 & $0,8 \%$ & $97,7 \%$ \\
\hline PB & 4 & 2 & 6 & $0,8 \%$ & $98,5 \%$ \\
\hline $\mathrm{AL}$ & 1 & 2 & 3 & $0,4 \%$ & $98,9 \%$ \\
\hline ES & 1 & 2 & 3 & $0,4 \%$ & $99,3 \%$ \\
\hline RN & - & 2 & 2 & $0,3 \%$ & $99,6 \%$ \\
\hline MS & 1 & - & 1 & $0,1 \%$ & $99,7 \%$ \\
\hline PA & 1 & - & 1 & $0,1 \%$ & $99,9 \%$ \\
\hline SE & - & 1 & 1 & $0,1 \%$ & $100,0 \%$ \\
\hline
\end{tabular}

Formado por PUC-RJ, FGV-RJ e UFMG, o último agrupamento situado no centro à direita do gráfico, possui como principal fator discriminante a maior proporção de artigos teóricos no eixo temático de Estratégia em Organizações. Nesse conjunto, destaca-se a FGV-RJ com $47 \%$ de suas publicações enquadradas como estudos teóricos. Poder-se-ia especular que, por ser uma das instituições mais prolíficas da área de Administração Pública, a qual apresenta grande percentual de artigos teóricos (HOCAYEN-DASILVA; ROSSONI; FERREIRA JR, 2008), é provável que os mesmos autores tenham aproveitado a capacidade em desenvolver esse tipo de estudo, voltando-se para a área de Estratégia. A PUC-RJ apresentou também 
grande percentual de estudos empíricos quantitativos, destacando-se um pouco das demais, principalmente pelo trabalho do professor Jorge Fe rreira da Silva, um dos autores mais prolíficos da área de Estratégia. Vale destacar ainda que embora a FGV-SP não tenha sido discriminada nessa análise, é a terceira em volume de artigos teóricos produzidos na área, superior até mesmo ao da UFMG, que integra esse grupo.

As informações resultantes da análise de correspondência possibilitaram a discriminação de grupos de instituições com base na similaridade apresentada por elas quanto à proporção de artigos orientados por eixo temático, tipo e método de pesquisa. No entanto não se buscou identificar nenhum tipo de relação entre as instituições que pudesse explicar as razões associadas a essa distribuição, o que desviaria do escopo deste trabalho. Porém, em conformidade com Rossoni (2006), acredita-se que a definição de eixos temáticos e de métodos de pesquisa seja influenciada por aspectos em níveis de análise mais específicos do que o nível organizacional, de modo que um importante elemento de homogeneização de métodos e temáticas na área de Estratégia de Organizações se dá por meio de pequenos agrupamentos de pesquisadores, que estão internamente mais relacionados.

Por fim, uma última análise foi realizada com a intenção de avaliar a distribuição regional da produção em Estratégia. Primeiramente, agregando-se as instituições com base em seu Estado (UF) de origem e realizando a contagem por período, foi possível perceber que a produção científica na área também é centralizada em poucas unidades da federação. Como exposto na Tabela 6, instituições localizadas no Estado de São Paulo representam 25,5\% do número de artigos publicados. Boa parte desse percentual, 66,15\% (129 de 195 artigos), está vinculada a somente três instituições: FGV-SP, USP e Mackenzie, três das mais prolíficas do país. Os Estados de Minas Gerais, Paraná, Rio de Janeiro e Rio Grande do Sul vêm na seqüência, com cerca de $12 \%$ das publicações cada um. Fica evidente também a representatividade das regiões sul e sudeste, pois nelas estão concentrados os Estados mais prolíficos, alcançando $82 \%$ de toda a produção na área. Como a maior parte dos programas de pós-graduação em Administração está localizada nessas duas regiões era esperado que refletisse na produção por unidade federativa. Por conta disso, no entanto, a discussão das temáticas em Estratégia tende a se concentrar na análise de fenômenos organizacionais dessas regiões, de modo que haja pouco espaço para o debate da Estratégia em Organizações localizadas nas regiões Norte, Nordeste e Centro-Oeste.

Essa concentração vem aumentando anualmente e, desde 2003, aponta um grau ainda maior em favor dos Estados da região sudeste, em especial São Paulo, em detrimento do sul, principalmente do Paraná e Rio Grande do Sul que não acompanharam o crescimento da área como um todo. Atualmente, a região sudeste já concentra $50 \%$ da produção sobre Estratégia. Destaca-se, ainda, a crescente presença de publicações originárias do Estado do Ceará nesse cenário. Além disso, a distribuição de artigos publicados por unidades federativas ao longo dos anos não sofreu impacto significativo derivado da escolha do local de realização dos eventos, salvo o 3Es de 2005, cuja realização no Estado do Rio de Janeiro parece ter influenciado, embora não significativamente, o aumento da concentração de publicações nesse ano pelas escolas localizadas nos Estados do Sudeste.

\section{Conclusões}

Ao longo deste estudo, constatou-se crescimento da área de Estratégia tanto com relação à quantidade de artigos publicados quanto no número de autores e que atuam na produção de trabalhos. Parte desse crescimento decorre da criação dos eixos temáticos de Gestão Internacional e Empreendedorismo, atraindo novos trabalhos para a discussão de assuntos nesses âmbitos. Por outro lado, não há como ignorar o efeito do 3Es enquanto encontro setorial criado especificamente para contribuir com a expansão e desenvolvimento das pesquisas em Estratégia no Brasil.

Com relação ao delineamento dos estudos da área, o que se percebeu foi a predominância de trabalhos empíricos amparados sobre métodos qualitativos de pesquisa, especialmente estudos de caso. Ao longo do período analisado, no entanto, foi possível notar que estudos quantitativos vêm ampliando espaço, principalmente por meio de análises do tipo survey e outros tratamentos estatísticos robustos para tratamento dos dados. Paradoxalmente, o crescimento de estudos quantitativos e o apego das pesquisas qualitativas a estudos de caso, somados ao baixo índice de pesquisas multimétodos e ensaios teóricos, suscitam reflexão sobre a maturidade da área. Por um lado, podem representar, respectivamente, preocupação além do aspecto exploratório e aproximação com a realidade organizacional, mas sob outra ótica, podem indicar fragilidade da pesquisa por recaírem em falácia epistemológica ou revelarem pouco 
esforço em transferibilidade, atendo-se mais à apresentação de casos do que à acumulação do conhecimento, como já haviam alertado Bertero, Vasconcelos e Binder (2003). Nesse ponto, por mais de $60 \%$ dos trabalhos terem optado por estudos de caso ou surveys, pode-se questionar ainda se os trabalhos decorrem da escolha do problema ou da pré-definição da estratégia de pesquisa. Até que ponto isso ocorre e se sofre influência da estrutura de fomento à publicação promovida pelos programas de pós-graduação e outros agentes institucionais como Capes ou, ainda, se é reflexo da existência de "tradições" de pesquisa na área, são questões a serem discutidas em novos estudos.

A análise de produção revelou ainda que, embora em escala menor do que no passado, a área apresenta pequeno número de instituições e unidades federativas produzindo a maior parte dos trabalhos. Essa estratificação indicou concentração da produção em Estados da região sul e sudeste (exceto o Espírito Santo), estando as instituições mais prolíficas localizadas no Estado de São Paulo que, curiosamente, responde por apenas pequena parcela dos estudos teóricos, orientando-se mais para artigos empíricos. Tal constatação leva a crer que a agenda de pesquisa em Estratégia privilegia fenômenos organizacionais dessas regiões, deixando em segundo plano problemas que ultrapassem esses limites regionais.

Os dados analisados neste trabalho possibilitam refletir também a respeito dessa participação do 3Es sobre a área de produção acadêmica em Estratégia. De acordo com Fachin (2006, p. 135-136), o 3Es tem como finalidade:

1) promover o encontro de pesquisadores [ ...] a fim de fomentar debates relevantes e estabelecer potenciais agendas de pesquisa; 2) promover o desenvolvimento da área de Estratégia no Brasil por meio do intercâmbio de informações sobre pesquisas que estão sendo realizadas nas instituições brasileiras; e 3) contribuir com o desenvolvimento das organizações no Brasil [ ...] por meio da disseminação de resultados das pesquisas e do intercâmbio mais próximo entre pesquisadores, profissionais e praticantes.

Amplamente entendido, o 3Es, assim como outros eventos, apresentam-se como espaço de divulgação e por ela ser sua natureza amplia o espaço de comunicação e debate acadêmico, como pode ser visto no seu impacto sobre o volume de artigos publicados. Mais do que isso, os dados apontam sua influência com relação ao número de participantes na área. Por ser canal de publicação, novos autores visualizam a possibilidade de expor suas pesquisas e acabam permanecendo no campo nos períodos seguintes.

Entretanto, mais relevante parece ser sua participação no debate teórico sobre Estratégia, concentrando mais de $60 \%$ desse tipo de produção, o que revela a importância do encontro, seja na sistematização do conhecimento desenvolvido na área, seja no direcionamento de agenda para futuras pesquisas sustentadas por diferentes quadros teóricos.

Outro aspecto indicativo de sua importância no desenvolvimento da área diz respeito à promoção da cooperação entre pesquisadores e à maior dispersão geográfica da produção, já que foi possível perceber aumento significativo do componente principal da rede de autores, além da menor concentração de publicação decorrente desse grupo, valendo essa observação também para instituições e unidades da federação.

Embora esses apontamentos propendam em favor da efetiva atuação do 3Es com relação aos objetivos que thes foram atribuídos pela ANPAD, as pesquisas devem ser lidas como um convite a mais aprofundamento em expor detalhes acerca do panorama apresentado. Além disso, os dados aqui dispostos consideraram apenas congressos, não incluindo periódicos ou outras formas de produção científica, não explorando também outros fatores com potencial influência sobre os dados analisados, como, por exemplo, o número de programas de pós-graduação, bem como suas diretrizes para a produção acadêmica, ou políticas de regulação institucional advindas da Capes. De qualquer modo, os resultados dispostos neste artigo apresentam aspectos relevantes que descrevem o comportamento da área nos últimos anos, apontando tendências e levantando questões sobre seu futuro, de modo que se acredita ter contribuído com reflexões acerca de seu desenvolvimento, especialmente no direcionamento dos próximos congressos e encontros temáticos.

\section{Notas}

1- Neste trabalho os termos Estratégia e Estratégia em Organizações foram utilizados de forma intercambiada para designar a área.

2- Para as análises por eixo temático, classificaram-se os artigos publicados nos 3 Es conforme as áreas 
temáticas da Divisão Acadêmica de Estratégia em Organizações do EnANPAD de 2006. Foram descartados quatro artigos da edição de 2003 e dois da edição de 2005 do 3Es, por serem considerados não alinhados à área.

3- Componentes são sub-redes cujos nós estão conectados entre si (DE NOOY; MRVAR; BATAGEL), 2005).

\section{Referências}

ANPAD. Anais do XXX Encontro Nacional da ANPAD. Rio de Janeiro: ANPAD, 2006.

BERTERO, C. O.; CALDAS, M. P.; WOOD JR, T. Produção científica em administração de empresas: Provocações, insinuações e contribuições para um debate local. In: EnANPAD, 27, 1998, Foz do Iguaçu. Anais... Rio de Janeiro: ANPAD, 1998.

BERTERO, C. O.; KEINERT, T. M. M. A evolução da análise organizacional no Brasil (1961-93), Revista de Administração de Empresas, v. 34, n. 3, p. 81-90, Maio/Jun. 1994.

BERTERO, C. O.; VASCONCELOS, F. C.; BINDER, M. P. Estratégia empresarial: a produção científica brasileira entre 1991 e 2002. Revista de Administração de Empresas, v. 43, n. 4, p. 48-63, Out./Dez. 2003.

BIGNETTI, L. P.; PAIVA, E. L. Ora (direis) ouvir estrelas: estudo das citações de autores de estratégia na produção acadêmica brasileira. Revista de Administração Contemporânea, v. 6, n. 1, p. 105-125, Jan./Abr. 2002.

BRAUN, T.; GLÄNZEL, W.; SCHUBERT, A. Publication and cooperation patterns of the authors of neuroscience journals. Scientometrics, v. 51, n. 3, p. 499-510, 2003. doi:10.1023/A:1019643002560

CALDAS, M. P.; TONELLI, M. J.; LACOMBE, B. M. B. Espelho, espelho meu: meta-estudo da produção científica em recursos humanos nos ENANPADs da década de 90. In: EnANPAD, 26, 2002, Salvador. Anais... Salvador: ANPAD, 2002.

CAMARGOS, M. A.; COUTINHO, E. S.; AMARAL, H. F. O perfil da área de finanças do EnANPAD: um levantamento da produção científica e de suas tendências entre 2000-2004. In: EnANPAD, 29, 2005, Brasília. Anais... Brasília: ANPAD, 2005.

CARVALHO, H. Análise multivariada de dados qualitativos: Utilização da HOMALS com o SPSS. Lisboa: Edições Silabo, 2004.

DE NOOY, W.; MRVAR, A.; BATAGEL, V. Exploratory Social Network Analysis with Pajek. New York: Cambridge University Press, 2005.

FACHIN, R C. Construindo uma associação científica: Trinta Anos de ANPAD: memórias, registros, desafios. Porto Alegre, ANPAD, 2006.

FLEURY, S. (Coord.) Análise do perfil dos artigos publicados na Revista de Administração Pública no período 1992-2002. Rio de Janeiro: EAESP/FGV, 2003.

FROEMMING, L. M. S.; LUCE, F. B.; PERIN, M. G.; SAMPAIO, C. H.; BEBER, S. J. N.; TREZ, G. Análise da qualidade dos artigos científicos da área de marketing do Brasil: as pesquisas survey na década de 90. Revista de Administração Contemporânea, v. 4, n. 3, p. 201-219, Set./Dez. 2000.

GUIMARÃES, T. B. C. Análise epistemológica do campo do empreendedorismo. In: EnANPAD, 28, 2004, Curitiba. Anais..., Curitiba: ANPAD, 2004.

HAIR, J.; ANDERSON, R. E.; TATHAM, R. L.; BLACK W. C. Análise multivariada de dados. Porto Alegre: Bookman, 2005.

HOCAYEN-DA-SILVA, A. J.; ROSSONI, L.; FERREIRA JR, I. Administração Pública e Gestão Social: a produção científica brasileira entre 2000 e 2005. Revista de Administração Pública, v. 42, n. 4, p. 655-680, jul./ago. 2008.

HOPPEN, N.; AUDY, J. L. N.; ZANELA, A. I. C.; CANDOTTI, C. T.; SANTOS, A. M.; SCHEID, R.; PERIN, M. G.; MECCA, M. S.; PETRINI, M. Sistemas de informação no Brasil: uma análise dos artigos científicos dos anos 90. In: EnANPAD, 22., 1998, Foz do Iguaçu. Anais... Foz do Iguaçu: ANPAD, 1998.

HOPPEN, N.; MEIRELLES, F. S. Sistemas de informação: um panorama da pesquisa científica entre 1990 e 2003. Revista de Administração de Empresas, v. 45, n. 1, p. 24-35, Jan./Mar. 2005.

MACHADO-DA-SILVA, C. L.; CUNHA, V. C.; AMBONI, N. Organizações: o estado da arte da produção acadêmica no Brasil. In: EnANPAD, 14, 1990, Belo Horizonte. Anais..., Belo Horizonte: ANPAD, 1990. 
MACHADO-DA-SILVA, C. L.; ROSSONI, L. Persistência e mudança de temas na estruturação do campo científico da estratégia em organizações no Brasil. Revista de Administração Contemporânea, v. 11, n. 4, p. 33-58, Out./Dez. 2007.

MACHADO-DA-SILVA, C. L.; VIZEU, F. Análise Institucional de Práticas Formais de Estratégia. Revista de Administração de Empresas, v. 47, n. 4, p. 89-100, Out./Dez. 2007.

MOODY, J. The structure of a social science collaboration network: disciplinary cohesion from 1963 to 1999. American Sociological Review, v. 69, p. 213-238, Apr. 2004. doi:10.1177/000312240406900204

MOREIRA, S. V. Análise documental como método e como técnica. In: DUARTE, J.; BARROS, A. (Org.). Métodos e técnicas de pesquisa em comunicação. São Paulo: Atlas, 2005. Cap. 17, p. 269-279.

NEUMAN, L. W. Social research methods: qualitative and quantitative approaches. Boston: Allyn \& Bacon, 1997.

PEGINO, P. M. F. As Bases filosóficas das publicações na área de estratégia das organizações nos Encontros Nacionais da ANPAD. In: EnANPAD, 29, 2005, Brasília. Anais Eletrônicos, Brasília: ANPAD, 2005.

PROENÇA, E. R.; LOPES, F. T. P.; MEUCCI, A. A construção do conhecimento em marketing: considerações sobre o discurso acadêmico e a produção científica do I Encontro de Marketing da ANPAD. In: EnANPAD, 29, 2005, Brasília. Anais Eletrônicos, Brasília, 2005.

READER, D.; WATKINS, D. The social and collaborative nature of entrepreuneurship scholarship: a cocitation and perceptual analysis. Entrepreneurship Theory and Practice, v. 30, n. 3, p. 417-441, May 2006. doi:10.1111/j.1540-6520.2006.00127.x

RODRIGUES, S. B.; CARRIERI, A. P. A tradição anglo-saxônica nos estudos organizacionais brasileiros. Revista de Administração Contemporânea, Edição Especial, p. 81-102, 2001.

ROSSONI, L. A dinâmica de relações no campo da pesquisa em organizações e estratégia no Brasil: uma análise institucional. Curitiba: UFPR, 2006. 296 p. Dissertação (Mestrado em Administração) - Programa de Pós-Graduação em Administração da Universidade Federal do Paraná, Curitiba, 2006.

ROSSONI, L.; GUARIDO FILHO, E. R. Cooperação interinstitucional no campo da pesquisa em estratégia. Revista de Administração de Empresas, v. 47, n. 4, p. 72-86, Out./Dez. 2007.

ROSSONI, L.; FERREIRA JR, I.; HOCAYEN-DA-SILVA, A. J. Administração de ciência e tecnologia: a produção científica brasileira entre 2000 e 2005. Revista de Administração da Unimep, v. 7, n. 3, p. 1-23, 2009.

SELLTIZ; JAHODA; DEUTSCH; COOK. Métodos de pesquisa nas relações sociais. São Paulo: EDUSP, 1974.

TONELLI, M. J.; CALDAS, M. P.; LACOMBE, B. M. B.; TINOCO, T. Produção acadêmica em recursos humanos no Brasil: 1991-2000. Revista de Administração de Empresas, v. 43, n. 1, p. 105-122, Jan./Mar. 2003.

VERGARA, S. C.; CARVALHO JR, D. de S. Nacionalidade dos autores referenciados na literatura brasileira sobre organizações. In: EnANPAD, 19, 1995, João Pessoa. Anais..., João Pessoa: ANPAD, 1995.

VIEIRA, F. G. D. Narciso sem espelho: a publicação brasileira de marketing, Revista de Administração de Empresas, v. 43, n. 1, p. 81-90, Jan./Mar. 2003. 